\title{
¿Para qué la educación no formal?: lo que piensan las familias de la experiencia en "La Otra Educación"1
}

\author{
Carlos Pizarro Cabrera ${ }^{2}$
}

\section{RESUMEN}

El neoliberalismo en Chile introdujo la lógica de mercado en todas las esferas, alterando la vinculación de las personas con sus derechos sociales, estableciendo dinámicas clientelistas propias de este fenómeno. Para las familias, la educación es una dimensión importante, cuya significación social está fuertemente influenciada por el espíritu neoliberal, que le da importancia económica por sobre otros factores, como los contenidos impartidos o los valores que entrega. A pesar de lo anterior, hay grupos y organizaciones sociales que buscan generar una dinámica distinta, para que la educación responda a los derechos de las personas y no sea funcional al mercado, generando instancias de resistencia y concientización. Este artículo da cuenta de lo que piensa un grupo de familias sobre la educación no formal, refiriéndose a la participación que tienen los niños, niñas y jóvenes en la intervención social que realiza la organización "La Otra Educación".

Palabras clave: educación no formal, familias, neoliberalismo, intervención social

\section{Why Non-Formal Education?: Families' Perspectives on "The Other Education" Experience}

\section{ABSTRACT}

Neoliberalism in Chile introduced a market logic in all spheres, altering people's connection to their social rights and establishing the consumerist dynamics inherent to this phenomenon. For families, education is an important dimension whose social significance is strongly influenced by the neoliberalist spirit, which prioritizes the economic importance of education over other factors, such as the contents taught or the values instilled. Notwithstanding this trend, there are social groups and organizations which seek to generate a different dynamic so that education responds to people's rights and not merely to their functional purpose in the market, thus

Artículo recibido 25/11/2018. Artículo aprobado el 25/12/2018.

2 Chileno. Trabajador Social. Coordinación Equipo Parlamentario H. Diputada

Claudia Mix Jiménez - Jefe de Gabinete. E-mail: cpc1989@gmail.com 
generating opportunities for resistance and awareness. This articles explores the opinions of a group of families on non-formal education, with respect the children and youth's participation in the social intervention carried out by the organization "La Otra Educación," (in English, "The Other Education").

Keywords: non-formal education, families, neoliberalism, social intervention

\section{Para que a educação não formal?: O que pensam as famílias da experiência em "A Outra Educação"}

\section{RESUMO}

O neoliberalismo no Chile introduziu a lógica de mercado em todas as esferas, alterando a vinculação das personas com seus direitos sociais, estabelecendo dinâmicas clientelistas próprias deste fenômeno. Para as famílias a educação é uma dimensão importante, cuja significação social está fortemente influenciada pelo espírito neoliberal, que lhe dá importância econômica por sobre outros fatores, como os conteúdos ministrados ou os valores que entrega. Apesar do anterior, há grupos e organizações sociais que buscam gerar uma dinâmica diferente para que a educação responda aos direitos das pessoas, e não seja funcional ao mercado, gerando instâncias de resistência e conscientização. Este artigo dá visibilidade ao que pensa um grupo de famílias sobre a educação não formal, referindo-se à participação que têm as crianças e jovens na intervenção social que realiza a organização "A Outra Educação".

Palavras-chave: educação não formal, famílias, neoliberalismo, intervenção social

\section{Antecedentes}

La importancia que le asignan todos los sectores de la sociedad a la educación es algo históricamente recurrente, más allá de los usos que le den. Es más, hoy es considerada un derecho humano fundamental, básicamente por las funciones que se le atribuyen. La ONU, en el artículo 26 de la declaración de los Derechos Humanos recalca que el objetivo que tiene la educación es el mantenimiento de la paz, ya que el desarrollo de la personalidad humana, el fortalecimiento de las libertades y el respeto a los derechos humanos ayudarán en la misión que tiene dicha institución, que es mantener el entendimiento de todos los pueblos y países (Organización de las Naciones Unidas, 2011). Por otra parte, la Comisión Económica para América Latina y el Caribe (CEPAL) identi- 
fica que ésta sirve para ayudar a la formación del Estado-Nación, a través de la instrucción de las generaciones jóvenes con todos los componentes necesarios (CEPAL, 2011), y el Programa de las Naciones Unidas para el Desarrollo (PNUD) ve en la educación el medio para que las personas tengan éxito en la sociedad y en el mundo laboral (PNUD, 2014). Es decir, si analizamos lo dicho, existe consenso sobre su relevancia y múltiples dimensiones.

Con la misma importancia que es tratada la educación en el mundo, es vista en Chile. Las familias la ven como el motor de superación de su situación socioeconómica, movilidad social y el camino para combatir la desigualdad. Por su parte, el Estado reconoce tres tipos de educación: formal, no formal e informal, donde solo pone esfuerzos y elabora políticas públicas para la primera, y básicamente legisla para el sistema educativo formal. Entre las medidas que ha tomado a este respecto está la temprana obligatoriedad de la instrucción primaria, la que al 2015 alcanzaba el 92,9\% de matrículas, y de secundaria, con una cobertura del $87,1 \%$, y una tasa de alfabetización del 99,4\%, según datos de la CEPAL. No obstante, quedan pendientes la calidad de los contenidos que se imparten en el sistema educativo, aspectos relativos a la infraestructura, carrera docente, temáticas de género, entre otras.

Durante los últimos años, en Latinoamérica y nuestro país, el debate en materias educativas se agudizó, guiado por diversos movimientos sociales que buscaban incidir en la agenda pública e instalar el tema de la crisis en la calidad de la educación. Es así como la llamada revolución pingüina en 2006 y la reactivación del movimiento estudiantil en 2011 dejaron claro que los cambios en esta materia debían ser estructurales, para que todas las personas tuvieran acceso a la educación, pero no a cualquiera, sino que a una educación digna y de calidad, independientemente del estrato social del que viniesen. Lo anterior debido a que el sistema actual se sustenta en “(...) una brecha importante en el acceso y calidad por decil de ingreso autónomo del hogar del cual proviene el estudiante" (PNUD, 2014, p. 173), es decir, un sistema educativo al servicio de la desigualdad. 
Favorecidas por estas movilizaciones, las políticas públicas en educación se transformaron en puntos programáticos importantes de los gobiernos, que modificaron algunas leyes inspiradas en la Constitución del ochenta, como lo fue la "Ley Orgánica Constitucional de Enseñanza", LOCE, dando paso a la "Ley General de Educación", LGE ${ }^{3}$, cuyos principios y fines apelan a elementos valóricos vinculados a conceptos como el respeto, la libertad y la diversidad, buscando incidir en la capacitación de las personas y aportar al desarrollo del país (Ley No 18.962, 2008).

No obstante, estos cambios no lograron dar respuesta a las demandas expresadas en las calles, manteniéndose la crisis de la educación, expresada en agudas diferencias de acceso y calidad de unos y otros. Las reformas no resultaron del todo eficaces y se profundizó el problema de la educación, manteniéndose su funcionalidad al sistema neoliberal (Redondo, 2015).

Recordemos que esta situación tiene su origen en las reformas neoliberales de la dictadura militar, lideradas por los "Chicago Boys", quienes impusieron la lógica del mercado en todos los aspectos del funcionamiento social, entre ellos, la educación, sustituyendo propuestas inclusivas y de acceso universal en esta materia como las formuladas por el presidente Salvador Allende (Comblin, 2001; Pinol, 2015). Todos estos cambios tuvieron un gran impacto social y cultural, afectando las relaciones interpersonales y superponiendo la figura del consumidor a la del ciudadano sin derechos ni protección, como efecto de la omnipresencia del mercado (Rojas, 2012).

No es extraño, entonces, sostener que las brechas en materia de educación son funcionales al sistema neoliberal, en la medida en que son causa y consecuencia de la desigualdad y refuerzan la segmentación social ya profundizada por la política económica (Sa-

Ley $\mathrm{N}^{\circ}$ 18.962. Dicha ley fue publicada un día antes que se hiciera el cambio de mando de la dictadura de Pinochet al Presidente Aylwin.

Ley $\mathrm{N}^{\circ} 20.370$. Surge como resultado de las movilizaciones sociales de los estudiantes secundarios durante el año 2006. 
der, 2005). Tampoco se puede obviar el descontento de la gente en este aspecto: "la indignación con el sistema educacional heredado y con el 'modelo neoliberal a la chilena' (...) que está demandando un verdadero cambio hacia más democracia y participación" (Redondo, 2015, p. 370).

Es así como aparecen movimientos sociales y ciudadanos que materializan su descontento en formas de organización, participación y resistencia, siendo el derecho a la educación una expresión más de este fenómeno. Diversos grupos comparten el diagnóstico planteado anteriormente y deciden elaborar un discurso contrahegemónico, que se traduce en prácticas y metodologías para hacer frente a la lógica individualista y de mercado que plantea la educación formal; diseñando opciones y/o complementos que fomenten la construcción de una sociedad distinta, dejando el valor del mercado de lado; superando la ausencia de igualdad de oportunidades al alero de pedagogías libertarias, la educación popular y otros movimientos fuera del sistema educativo hegemónico, y que están relacionados a la educación no formal (Lamata et al., 2003). Estos buscan entregar herramientas a los sectores populares y vulnerados, y si bien se apoyan en la enseñanza de materias que imparte la educación formal, también las complejizan. Estas agrupaciones $y / u$ organizaciones aspiran a mayor libertad, autonomía social y política, mediante la búsqueda del sentido de la dignidad y confianza en quienes participan de ellas y en sus propuestas (Torres, 2002).

"La Otra Educación" es una de estas tantas manifestaciones. Se trata de una agrupación de jóvenes chilenos que levantan una red de escuelas libres con presencia en comunas de la Región Metropolitana y la ciudad de Puerto Montt. Se caracterizan por trabajar con niñas, niños y jóvenes (NNJ), familias y comunidades de los sectores más vulnerados de la población, desde hace siete años. Estos espacios fomentan procesos educativos diferentes, aspirando a nuevas lógicas de relaciones humanas igualitarias, implementando una intervención multidisciplinaria con enfoque de derechos, aspirando y promulgando una serie de va- 
lores contrarios al neoliberalismo, como son la solidaridad, el trabajo en equipo, la afectividad, entre otros. Entre sus espacios educativos históricos de destaca la Escuela Libre La Chascona, ubicada en la comuna de la Granja, en la población Poeta Neruda (La Otra Educación, 2016).

La investigación sobre los métodos educativos no formales no es muy común debido a la complejidad en la definición de los procesos (Mejía, 2013; López y Quiroz, 2014; Suárez, et al., 2015). No obstante, el proyecto "La Otra Educación" ofrece una interesante posibilidad de profundizar este aspecto (González y Ramos, 2013; Guzmán y González, 2015; Garrido, et al., 2016), a condición de que se abordara su relación con la intervención social y los significados que las propias familias involucradas le dan al proyecto. A partir de ello, se llevó a cabo un estudio que contempla la visión de estas familias en el proceso y la relación que mantienen con las escuelas libres, donde participan niños, niñas y jóvenes (NNJ). La pregunta de investigación se orientó a conocer los significados atribuidos por las familias a la participación de niños, niñas y jóvenes de la Escuela Libre de La Granja en La Otra Educación.

\section{Metodología}

El proceso metodológico tuvo como referente el paradigma interpretativo en el contexto de un enfoque cualitativo, cuyo propósito fue comprender los significados atribuidos por las familias a la participación de los niños, niñas y jóvenes (NNJ) en el proyecto de educación no formal (ENF) de la escuela libre La Chascona, rescatando sus expectativas y todo lo que involucra relacionalmente a las personas (Strauss y Corbin, 2002). De esta manera, se identificaron elementos expresados en sus relatos que después fueron analizados e interpretados, ordenando las textualidades de acuerdo con temas comunes, destacando las citas con mayor representatividad. Del total de personas entrevistadas, solo dos padres participaron, siendo las madres quienes presentaron mayor disposición. Se trató de un estudio de caso de tipo exploratorio, en vistas a profundizar en las características de un fenómeno emergente, 
a saber, el impacto de la educación no formal en las familias que asisten al programa.

Para la recolección de información se emplearon, como técnicas, el análisis documental y la entrevista semiestructurada, construida previa categorización de los aspectos sobre los cuales se deseaba consultar a las familias. Las dimensiones fueron el nivel socioeconómico, aspectos sociorelacionales y la participación de las familias en el proyecto, y guiaron la confección de preguntas. Este instrumento fue revisado por un equipo interdisciplinario de ciencias sociales y educación.

La muestra, de tipo intencionada, se extrajo del conjunto de familias de NNJ que participaron en la Escuela Libre de La Otra Educación ubicada en la comuna de La Granja, entre 2016 y 2017, en función de, entre otros criterios, su disposición a participar en el estudio (voluntariedad) y la accesibilidad del equipo. Antes de la selección definitiva, se consultó a informantes clave y representativos del proyecto. Los primeros fueron las familias que participaban activamente del proyecto o mostraron algún tipo de vinculación con el mismo. También se estimó importante que sus hijos y/o hijas estuviesen desde el inicio en la implementación del proyecto. En cuanto a los informantes representativos, se trató de personas que pertenecieran al territorio donde se localizó el estudio y que participaran en la escuela libre. Para garantizar la validez y confiabilidad de la información, se triangularon técnicas e informantes y se desarrolló un proceso de análisis permanente de los datos que iban siendo obtenidos. El método de análisis escogido fue el de comparación constante, MCC, basado en la teoría fundamentada. Ello permite ampliar el espectro del análisis hacia contextos similares, es decir, hacia otros lugares donde se está desarrollando esta experiencia.

\section{Análisis de la información}

El análisis de la información permitió trabajar sobre las dimensiones preestablecidas y las categorías respectivas ligadas a los objetivos de esta investigación; estas son, las socioeconómicas, las 
sociorelacionales y el nivel de participación. Todas estas dimensiones fueron exploradas a través de los discursos de las madres y padres entrevistados, relevando la importancia de la subjetividad.

En la dimensión socioeconómica se presentaron temas importantes para las familias como el significado que atribuyen a la educación, en especial a la de carácter formal. Aquí se observa fuertemente la influencia del mercado, en la medida en que la perciben como un "servicio" cuya calidad varía en función de los recursos económicos que se posean, lo que genera desigualdad, exclusión y la percepción que se trata de una inversión que implica un alto costo. También la educación se presenta como una posibilidad de movilidad social, en la que los adultos tienen una especie de "fe inquebrantable". Si sus hijos se educan bien "serán algo en la vida", lo que refleja la sobrevaloración de la educación formal pues afectaría todos los aspectos de la vida de las personas.

En la dimensión sociorelacional se identificó que no existe una definición uniforme entre las familias sobre qué es la educación. Esta estaría determinada por el contexto y por el periodo histórico. Donde sí hubo consenso es en la vinculación inmediata entre educación y "colegio", es decir, la asociación con la educación formal. En relación con el para qué de la educación, se presentan respuestas interesantes. La mayoría de las familias la ven como una "revancha personal" que les permite dejar atrás sus frustraciones. Por ello, hacen todo lo posible para que sus hijos tengan "mejores oportunidades”, viendo en la educación la mejor herencia y/o inversión.

La educación no formal y, en específico, "La Otra Educación", es un espacio muy valorado por el apoyo académico que presta a quienes participan de dicho proyecto. Asimismo, se valora el acompañamiento permanente y personalizado que significa adaptarse al nivel de desarrollo de cada niño o niña, no siendo ello necesariamente un objetivo del proyecto "La Otra Educación". También se reconocen los aportes que el proyecto hace en aspectos sociales, en la medida en que potencia habilidades y refuerza hábitos para 
el desarrollo del niño/a y de su entorno, lo que enriquece y retroalimenta la intervención. Junto con estas valoraciones positivas, la mayoría de las familias no entiende que los conflictos que se generan entre voluntarios/as y niños/as se manejen en forma simétrica, pues sus pautas de crianza obedecen a lógicas tradicionales, caracterizadas por relaciones jerárquicas.

En cuanto a la participación, se evidenció que, aunque este tipo de proyectos busque promoverla e incentivarla, los resultados no son los esperados. Las familias se vinculan poco con el proyecto, lo conocen superficialmente, siendo sus hijos/as los principales canales de información. Posiblemente ello sea atribuible al hecho de que la propuesta potencia el compromiso desde una perspectiva distinta a la de la educación formal, particularmente en lo referido al trabajo personalizado y a la excusión de la lógica económica. Pese a ello, las familias valoran positivamente el espacio y lo identifican como inusual con relación a los modos habituales de funcionamiento de los sistemas de enseñanza, destacando lo que perciben como un "trato humanitario".

Por último, un hallazgo relevante fue la alta valoración que le otorgan al uso de los espacios públicos, como la sede de la Junta de Vecinos en donde se realizan las jornadas.

\section{Conclusiones y reflexiones}

Hoy nos encontramos en una situación social compleja, por el carácter sistémico y global de su origen. El neoliberalismo implantó la cultura del mercado, condicionando las relaciones sociales a lógicas del consumo. Se trata de dinámicas y estilos de relación que se naturalizan, así como también se naturaliza la desprotección y las dificultades de satisfacer derechos fundamentales. Los oprimidos, en el decir de Paulo Freire, no tienen posibilidades de escoger, pues son los "opresores", la élite política y económica, quienes definen los límites de nuestras acciones. Ello se ve facilitado por la fragmentación del mundo social, la individualización de los comportamientos como estrategia que permite, al menos, garantizar la sobrevivencia. 
Desde la imposición de este modelo, los derechos fundamentales han estado condicionados a la voluntad de las autoridades políticas, que niegan o permiten a la ciudadanía un acceso restringido y limitado a su satisfacción. Derechos como la atención en salud, la vivienda, la previsión social, la educación, se transformaron en bienes de consumo.

Otro elemento que cabe destacar es que las familias que participaron del estudio tienen distintas percepciones de lo que es la educación: las vivencias personales y factores culturales dominantes juegan en ello un rol fundamental. Pese a esto, la mayoría estima que se trata de un aspecto altamente valorado y un factor crucial en la búsqueda de mejores oportunidades, una estrategia para la superación de la pobreza y la movilidad social, lo que explica por qué se adaptan a las exigencias y mecanismos que impone la sociedad de mercado para su acceso a ella.

Este aspecto tiene un efecto negativo sobre la visibilización y valoración de modos alternativos de desarrollar procesos de enseñanza/aprendizaje de carácter no formal, como los que plantea este proyecto.

La marginalidad de los movimientos educativos contrahegemónicos y de la educación no formal no es casual. Sus objetivos no obedecen a una lógica mercantil, más bien se sitúan en posición crítica a ella, haciendo visibles los problemas que afectan a los excluidos. La liberación del oprimido, como plantea Freire, del pueblo, de la familia popular, es la razón porque la que nace y se mantiene este movimiento. La transformación cultural se vuelve entonces uno de los mayores desafíos que enfrenta nuestra sociedad contemporánea: cambiar la lógica de la educación como un bien de consumo a otra que la entienda como una herramienta y un espacio para el desarrollo individual y social. La educación no formal se plantea como una estrategia necesaria y útil para lograr este propósito. La importancia de espacios contrahegemónicos de formación alternativa es esencial para educar sobre la construcción de una sociedad más justa y equitativa. 
Ahora, respondiendo a los cuestionamientos que motivaron esta investigación, se pueden mencionar los siguientes aspectos subrayados por las familias:

- El valor de la educación formal. La significación atribuida por las familias va en dos direcciones; como elemento fundamental para el desarrollo de cualquier persona y como herramienta valórica fundamental para "hacer el bien".

- La educación es vista como un bien de consumo. Pese a haber sido repuesto en la escena nacional, a partir de las movilizaciones de 2006, su carácter de "derecho humano básico", las familias entrevistadas destacan que hay que "pagar para acceder a una educación de calidad" y que la calidad sigue estando sujeta a los mayores o menores recursos económicos de las familias.

- La invisibilización de la educación no formal, vista solo como dispersión y entretenimiento. A pesar de que identifican características positivas a las dinámicas y procesos que acompañan la educación no formal y reconocen el impacto positivo que tiene sobre sus hijos, no se apropian completamente del sentido y utilidad de la intervención social realizada por "La Otra Educación”, relegando el trabajo de este organismo a un segundo plano. El colegio parece imponerse como la única instancia educativa válida.

- El éxito en el desarrollo de metodologías alternativas para los NNI. La escuela libre impulsada por "La Otra Educación" ha logrado ser reconocida como un espacio protector, contenedor e inusual, en donde se promueve la libertad de expresión con márgenes de respeto y transversalidad entre las personas asistentes. También valoran el compromiso por parte de los/as voluntarias que apoyan social y académicamente los procesos de los NNJ.

- La baja participación de las familias en el proyecto. Como se indicó, el involucramiento de las familias es débil, limitando su cooperación a aportes económicos y/o de determinados servicios. La razón que aducen es la falta de tiempo, sus extensas 
jornadas laborales, contextos familiares complejos, escasa información respecto de aquellas actividades en las que pueden participar y la poca interacción que existe con los voluntarios/ as. En este punto se observa la reproducción de dinámicas propias de la educación formal. El contacto de las familias con el sistema educacional es de carácter informativo, restringido a reuniones de apoderados o citaciones puntuales.

- El aporte a la comunidad. Las familias detectaron que este tipo de proyectos no está en todas partes y por ello valoran su particularidad. Algunos advierten cambios notorios en el comportamiento de sus hijos/as, otros destacan el apoyo académico que se brinda, y otros simplemente aprecian la existencia de esta instancia y su mantención en el tiempo.

- La reinvención de la sede social y la utilización de los espacios públicos. El proyecto de "La Otra Educación" se desarrolla en espacios distintos a una escuela: iglesias, clubes deportivos, casas recuperadas, sedes sociales y juntas de vecinos (JJVV) son solicitados para su uso, siendo esta última la ocupada por el proyecto. Destinar este tipo de espacios para realizar trabajo social con NNJ genera una percepción positiva por parte de las familias y la comunidad en su conjunto. Las JJVV suelen ser ocupadas para el desarrollo de actividades con adultos mayores, dejando fuera otros grupos sociales y disminuyendo las posibilidades de integración comunitaria. Otra situación que cabe destacar es que los NNJ que participan en el proyecto utilizan de manera responsable estos espacios comunes, lo que contribuye a disminuir los prejuicios hacia ellos en tal sentido. Asimismo, se revitalizan espacios comunitarios como plazas y parques, que en su mayoría son usados para el consumo de alcohol y drogas.

- El rol de la familia popular en la educación. El sujeto popular, ha recibido el impacto de las transformaciones que han afectado a la sociedad en su conjunto. Entre dichos impactos está la tensión entre las posibilidades de acceso al consumo y el costo 
que las familias deben pagar por ello. Si bien no se trataba de familias pobres en el sentido clásico y restringido del término, sí se encontraban afectadas por privaciones en diferentes aspectos y grados.

Finalmente, en cuanto a la intervención social desde el trabajo social, se reafirma la importancia de esta profesión para el desarrollo de proyectos como el descrito, particularmente sus aportes a nivel de la diversidad de sus metodologías de trabajo. La profesión sigue estando del lado de los oprimidos, aportando, a pesar de las limitaciones del sistema en el que la misma profesión está inserta, una perspectiva dialógica y crítica, lo que es reconocido como un aporte por el proyecto que impulsa "La Otra Educación". Como se indicó, el sujeto popular ha recibido el impacto de fuertes transformaciones sociales que han modificado sus dinámicas familiares y ello debe ser considerado a la hora de intervenir. Hoy en día, la educación y la intervención social deben ser vistas como herramientas centrales para la lucha contra viejos y nuevos problemas sociales, tales como la violencia de género, el maltrato y la precariedad económica de los adultos mayores, la depredación del medio ambiente y la persecución a los movimientos ambientalistas, la criminalización de las comunidades indígenas, entre otras situaciones respecto de las cuales urge intervenir.

\section{Bibliografía}

Comblin, J. (2001). El Neoliberalismo: ideología dominante en el cambio de siglo. Santiago de Chile: Ediciones ChileAmérica CESOC.

Comisión Económica para América Latina y el Caribe. (2011). Educación, Desarrollo y Ciudadanía en América Latina. Propuestas para el debate. Santiago de Chile: CEPAL.

Comisión Económica para América Latina y el Caribe. (2018). Perfil Nacional Socio-demográfico. Recuperado de http:// interwp.cepal.org/cepalstat/Perfil_Nacional_Social. html? pais $=$ CHL\&idioma $=$ spanish

Garrido, A., González, C., Morales, M., Parra, J. y Quevedo, C. (2016). Modelo Educacional Alternativo: Investigación a través del significado de los participantes de la Escuela Libre "La 
Chascona". (Tesis para optar al grado de licenciado en Trabajo Social). Universidad Católica Silva Henríquez, Santiago de Chile.

Guzmán, F. y González, G. (2015). Autogestión para la Educación: Una alternativa a la Educación de Mercado. (Tesis para optar al título de periodista). Universidad de Santiago de Chile, Santiago de Chile.

González, R. y Ramos, P. (2013). La Otra Educación: Red de Escuelas Libres en Chile. Percepciones de educadores de la red de escuelas libres en Chile, en la región metropolitana, sobre el proyecto educativo libertario en el que participan. (Tesis para optar al título profesional de Educadoras de Párvulos y Educación Básica Inicial). Universidad de Chile, Santiago de Chile, Chile.

La Otra Educación (2016). Memoria Anual 2016. Documento de elaboración propia.

Lamata, R., Domínguez, R., Baráibar, J. M., Bonell, L., Casellas,L. y Gamonal, A. (2003). La construcción de procesos formativos en educación no formal. Madrid, España: Narcea.

La Otra Educación. (2016). Memoria anual.

Ley $N^{\circ}$ 18.962. Ley Orgánica Constitucional de Enseñanza. Recuperado de https://www.leychile.cl/Navegar?idNorma=30330

Ley N ${ }^{\circ} 20.370$. Establece la Ley General de Educación. Recuperado de http://www.leychile.cl/Navegar?idNorma=1006043

López, A. y Quiroz, E. (2014). Educación comunitaria: una propuesta alternativa para los pueblos indígenas de Oaxaca-México. Polis, 38. Recuperado de http://polis.revues.org/10107

Mejía, M. (2013). Educaciones y pedagogías críticas desde el Sur (cartografías de la educación popular). Chile: Quimantú.

Organización de las Naciones Unidas. (1948). Declaración Universal de los Derechos Humanos. United Nations.

Pinol, B. A. (Ed.). (2015). Democracia versus neoliberalismo: 25 años de neoliberalismo en Chile. Buenos Aires, Argentina: CLACSO.

Programa de las Naciones Unidas para el Desarrollo. (2014). Auditoría a la Democracia. Más y mejor democracia para un Chile inclusivo. Chile.

Redondo Rojo, J. (2015). La extinción de la educación pública en Chile. Buenos Aires, Argentina: CLACSO.

Rojas Hernández, J. (2012). Sociedad bloqueada: movimiento estudiantil, desigualdad y despertar de la sociedad chilena. Santiago de Chile: RIL Editores. 
Sader, E. (2005). La trama del neoliberalismo: mercado, crisis y exclusión social. Buenos Aires, Argentina: CLACSO.

Strauss, A. y Corbin, J. (2002). Bases de la investigación cualitativa. Técnicas y procedimientos para desarrollar la teoría fundamentada. Colombia: Universidad de Antioquia.

Suárez, D., Hillert, F., Ouviña, H. y Rigal, L. (2015). Pedagogías críticas en América Latina. Experiencias alternativas de educación popular. Buenos Aires, Argentina: Noveduc.

Torres, C. A. (2002). Paulo Freire y la agenda de la educación latinoamericana en el siglo XXI. Buenos Aires, Argentina: CLACSO. 